\section{Frame retractions so they hold firm}

The retraction last month (see Nature 512,338 ; 2014) of the paper 'Generation of pluripotent stem cells from adult human testis' by S. Conrad et al. (Nature $456,344-349 ; 2008$ ) has caused some confusion in the scientific community because of its ambiguous wording, which does not serve the purpose of formally amending the scientific record.

Pluripotency is a well-defined property of stem cells both in vivo and in vitro (see, for example, J. Nichols and A. Smith Cold Spring Harb. Perspect. Biol. 4, a008128; 2012). However, the retraction statement refers to the cells derived in the original paper as being "pluripotent to some level", which wrongly implies that there are different degrees of pluripotency. Such scientific sloppiness is misleading and runs counter to rigorous, evidencebased presentation of results (see, for example, Nature 510, 187-188; 2014 and E. Cattaneo and G. Corbellini Nature 510, 333-335; 2014) .

Furthermore, it is unclear what the statement "the original conclusions are not as robust as presented in the original paper" actually means - for example, it could imply that some or all of the earlier conclusions are not entirely invalidated. In which case, we think that those details should have been specified. Joachim Kirsch University of Heidelberg, Germany.

Hans Schöler Max Planck Institute for Molecular Biomedicine, Münster, Germany. joachim.kirsch@urz.uniheidelberg.de

\section{Pregnancy: study the mother's DNA as well}

Research into the effects of epigenetic changes during pregnancy on the mother's long-term health is almost nonexistent. This contrasts sharply with the wealth of attention paid to such cell-heritable changes, which alter gene activity but not DNA sequence, in the fetus and placenta as a developmental origin of health and disease (see S. S. Richardson Nature 512, 131-132; 2014).

The pregnant body undergoes huge changes: extensive tissue remodelling, expansion in blood volume by as much as $100 \%$, immunological and metabolic alterations, and extensive shifts in hormone signalling. And complications such as gestational diabetes and pre-eclampsia, which both subside after giving birth, are known to increase the mother's risk of later developing type 2 diabetes (L. Bellamy et al. Lancet 373, 1773-1779; 2009) or hypertension and stroke (L. Bellamy et al. Br. Med. J. 335, 974; 2007). These all have big implications for public health.

We need to proceed cautiously when building causal narratives for health outcomes, and it might be hard to study epigenetic effects in mothers when few other results are available for comparison. But grant applications, hypotheses and experimental design should not be framed by the fetus alone. Hannah Landecker University of California, Los Angeles, USA. landecker@soc.ucla.edu

\section{Pregnancy: no safe level of alcohol}

In our view, Sarah Richardson and colleagues understate the risks of alcohol consumption during pregnancy (Nature 512, 131-132; 2014). Fetal alcohol spectrum disorders are among the three leading causes of intellectual disability (C. O'Leary et al. Dev. Med. Child Neurol. 55, 271-277; 2013).

Alcohol can disrupt brain development throughout pregnancy, often without causing the recognizable facial changes of fetal alcohol syndrome. The child can experience life-long cognitive and behavioural effects as a result (see, for example,
S. N. Mattson et al. Neuropsychol. Rev. 21, 81-101; 2011).

A recent meta-analysis of 34 published cohort studies has revealed an association between moderate levels of alcohol exposure in utero and behavioural problems during childhood (A. L. Flak et al. Alcohol Clin. Exp. Res. 38, 214-226; 2014). The study authors conclude that there is no known safe amount of alcohol that can be consumed while pregnant.

Thoughtful discussion of the risks of drinking alcohol during pregnancy is likely to enhance, rather than restrain, women's freedom in the long term. Elizabeth R. Sowell University of Southern California; and Children's Hospital Los Angeles, California, USA.

esowell@chla.usc.edu

Michael E. Charness VA Boston

Healthcare System; Harvard

Medical School; and Boston

University School of Medicine, USA.

Edward P. Riley San Diego State

University, California, USA.

\section{Count the social cost of oil sands too}

Efforts to eliminate carbon pollution should not divert attention from other pressing issues that have accompanied oil-sands development (see W. J. Palen et al. Nature 510, 465-467; 2014), such as indigenous rights, health inequities and social problems. In Canada, for example, housing shortages, substance abuse and food insecurity have all been attributed to Alberta's large-scale oil-sands production.

Furthermore, halting production from oil sands will not solve climate or environmental problems at a stroke. In our view, a better approach would be to ban fuels that emit large amounts of carbon dioxide, sulphur dioxide and harmful gases. This moratorium might include fuels such as coal, lignite, shale gas, and oil from tar sands or shale (see also A. Leach and B. Boskovic
Nature 511, 534; 2014).

In summary, it is important for energy and environmental policies to be discussed alongside those that involve public health, sustainable economic development, job creation and social justice (see also T. Measham and D. Fleming Nature 510, 473; 2014).

Stephanie Montesanti* University of Calgary, Canada. srmontes@ucalgary.ca ${ }^{*}$ On behalf of 8 correspondents (see go.nature.com/ichex2 for full list).

\section{Consider human will in psychology studies}

To achieve the improvements advocated by Emily Holmes and colleagues for psychological treatments (Nature 511, 287-289; 2014), researchers need to conceptually link studies of specific psychiatric disorders with fundamental processes that are shared by different disorders.

Psychologists often manipulate the environment of study participants (the independent variable) to alter a person's response or behaviour (the dependent variable). For example, they might compare the effects of threatening or neutral images on a subject's physiological arousal or memory. This approach lends itself to statistical analysis of group data, but it overlooks the important point that humans already control their environment by altering their responses. An example would be an anxious person who actively seeks safety by avoiding eye contact.

Research methodologies need to take into account the fact that such negative-feedback mechanisms exert control at all levels, including physiological, psychological and social (see T. A. Carey Lancet 382, 1403-1404; 2013).

Warren Mansell University of

Manchester, UK.

Timothy A. Carey Centre for Remote Health, Alice Springs,

Australia.

warren.mansell@manchester.ac.uk 